\title{
Dietary role in the development and treatment of inflammatory bowel disease
}

Jae Young Kim, MD, PhD

Department of Pediatrics, Gyeongsang National University Changwon Hospital, Changwon, Korea

\section{Key message}

Although the precise pathogenesis of inflammatory bowel disease (IBD) is unclear, dietary factors seem to play a significant role. Dietary modifications including enteral nutrition and the Crohn disease exclusion, specific carbohydrate, and antiinflammatory diets show a potential ability to downregulate gut inflammation. These nutritional interventions have various degree of efficacies with limited side effects profile for treating pediatric IBD, but data from randomized prospective studies are lacking, and further studies are warranted.

Inflammatory bowel diseases (IBDs) are immune-related chronic intestinal inflammatory conditions including the two prototypes of Crohn disease (CD) and ulcerative colitis (UC). IBD except for the early-onset phenotype is a multifactorial disease with an unclear pathogenesis. Complex interactions between susceptible genes and one or more environmental factors are possible drivers in IBD development. ${ }^{1)}$ Genome-wide association studies and next-generation sequencing studies have identified over 200 genetic loci associated with $\mathrm{IBD}^{2,3)}$ that comprise $19 \%$ and $26 \%$ of the heritability for UC and CD, respectively. ${ }^{3)}$ Therefore, susceptibility genes cannot explain the worldwide increasing rate of IBD over time, especially in Western countries, East Asia, and many newly industrialized countries. ${ }^{1)}$ The human genetic constituents have barely changed during its long evolutional history. Thus, the increasing incidence of IBD seems to be more likely related with exposure to various environmental factors rather than genetic factors. ${ }^{1)}$ This is supported by epidemiologic observation data on immigrants from low incidence regions to high IBD incidence countries that the second generation of immigrants show a similar risk of IBD development as does the indigenous population. ${ }^{4)}$

Among the environmental factors, diet appears to play a plausible role in building the physiologic gut microenvironment and contributing to the development of IBD. In a review article, Park at al. described the dietary role as an environmental factor in developing inflammatory bowel disease. ${ }^{5)}$ Complex carbohydrates, fiber-rich vegetables, and fruits have beneficial impli- cations in gut homeostasis. ${ }^{1,6)}$ Short-chain fatty acids (SCFAs), mainly butyrate, which is produced from the bacterial fermentation of nondigestible carbohydrates or amino acid degradation, demonstrate an anti-inflammatory property through immunomodulation in both innate and adaptive immunity. ${ }^{6,7)}$ Butyrate also induces mucus production by stimulating the epithelial cells and rearrange tight junctions. ${ }^{7)}$ A systemic review showed that fiber- and fruit-rich diets were associated with a low risk of $\mathrm{CD}$, while a vegetable-rich diet was associated with a low risk of UC. ${ }^{8)}$ On the contrary, Westernized nutritional habits, including the consumption of high amounts of animal fat and refined sugar, low amounts of fiber, and the food additive carrageenan, have been suggested as possible triggers in IBD development. $\left.{ }^{6}\right)$ The Western diet (WD) may alter the composition and function of the gut microbiota (dysbiosis), which is associated with poor production of beneficial microbial metabolites such as SCFAs and destruction of the mucosal barrier due to expansion of colonic mucus-degrading bacteria. ${ }^{6,8)}$ The WD is also associated with facilitating production of pro-inflammatory cytokines and modulating intestinal permeability. ${ }^{6}$ Several epidemiologic studies have shown that a diet with high amounts of saturated fatty acids and animal proteins is linked to an increased risk of IBD. ${ }^{8)}$ Emulsifiers and artificial sweeteners induce chronic gut inflammation and weaken the gut barrier function. ${ }^{6}$ Although the role of diet in development and progression of IBD is not fully understood, it is obvious that chronic WD exposure can induce dysbiosis and low-grade gut inflammation. Dysbiosis has been documented in many IBD patients and is accepted as playing a key role in gut inflammation. ${ }^{6,8)}$ However, it remains unclear whether dysbiosis is a cause or a change as a consequence of IBD.

According to the evolving landscape of diet role for gut homeostasis, dietary therapies have been applied primarily in CD. Dietary interventions have some advantages including its physiologic property in remission induction through restoration of gut microbiota balance. With the introduction of biologics, the target of treatment has shifted from relieving symptoms to achieving mucosal healing using a target to target strategy. Park at al. showed recent data on the efficacy of dietary modification

\footnotetext{
Corresponding author: Jae Young Kim, MD, PhD. Division of Pediatric Gastroenterology and Hepatology, Department of Pediatrics, Gyeongsang National University Changwon Hospital, 11 Samjunga-ro, Sungsan-gu, Changwon 51472, Korea 凶E-mail: pedkim@gnuh.co.kr, http://orcid.org/0000-0001-8563-9740

Received: 10 January, 2020, Revised: 9 June, 2020, Accepted: 11 June, 2020

This is an open-access article distributed under the terms of the Creative Commons Attribution Non-Commercial License (http://creativecommons.org/licenses/bync/4.0/) which permits unrestricted non-commercial use, distribution, and reproduction in any medium, provided the original work is properly cited. Copyright (c) 2020 by The Korean Pediatric Society
} 
Table 1. Proposed dietary interventions to help control IBD in children and adolescents ${ }^{8-10}$

\begin{tabular}{|c|c|}
\hline Diet & Efficacy and limitation \\
\hline \multirow{3}{*}{$\begin{array}{l}\text { Exclusively enteral nutrition } \\
\text { (EEN) }\end{array}$} & Induction therapy in mild to moderate CD \\
\hline & Clinical improvement \\
\hline & Mucosal healing \\
\hline \multirow[t]{2}{*}{$\begin{array}{l}\text { CD exclusion diet plus PEN } \\
\text { (CDED plus PEN) }\end{array}$} & $\begin{array}{l}\text { Induction remission, sustained remission } \\
\text { in mild to moderate CD (limited data; fur- } \\
\text { ther studies needed) }\end{array}$ \\
\hline & Better tolerate than EEN \\
\hline \multirow[t]{4}{*}{ CD exclusion diet (CDED) } & Maintenance therapy \\
\hline & Clinical improvement (limited data) \\
\hline & No mucosal healing \\
\hline & Validation needed in children \\
\hline \multirow[t]{4}{*}{ Specific carbohydrate diet } & Maintenance therapy \\
\hline & Clinical improvement \\
\hline & No mucosal healing \\
\hline & Poor long-term adherence \\
\hline \multirow[t]{4}{*}{ CD-TREAT diet } & Maintenance therapy \\
\hline & Clinical improvement (limited data) \\
\hline & No mucosal healing \\
\hline & Superior tolerate than EEN \\
\hline \multirow[t]{4}{*}{ Anti-inflammatory diet } & Maintenance therapy \\
\hline & Clinical improvement (limited data) \\
\hline & Mucosal healing (further studies needed) \\
\hline & No current evidence in pediatric IBD \\
\hline
\end{tabular}

IBD, inflammatory bowel disease; CD, Crohn disease; PEN, partial enteral nutrition.

and modulation of intestinal microbiota in the biologic era. ${ }^{5)}$ Exclusive enteral nutrition (EEN), the first introduced dietary intervention, achieves mucosal healing and has similar remission rates to those of corticosteroid in cases of newly diagnosed as well as relapsed pediatric active luminal CD. ${ }^{6,9)}$ EEN improves endoscopic severity and histology, supports continuous growth, and minimizes side effects. $\left.{ }^{6}\right)$ EEN possibly influences the gut bacterial milieu, which results in downregulating immune activation by decreasing proinflammatory cytokines and restoring barrier integrity. ${ }^{9)}$ EEN has unsolved problems with low compliance due to its tasteless palatability and sparse long-term outcomes. To improve these problems, other various dietary interventions have been implicated. ${ }^{9-11)}$ A recent study showed that the CD exclusion diet plus partial enteral nutrition was effective for maintaining sustained remission in mild to moderate pediatric CD. ${ }^{10)}$ Other various dietary interventions including the specific carbohydrate diet, CD exclusion diet, CD-TREAT diet (solid food mimicking the composition and effects of enteral nutrition), and anti-inflammatory diet have been implemented to help IBD treatment with varying degrees of data quality in children. ${ }^{9,11)}$ The effects of these formulations are still being evaluated (Table 1). Such works may not only lead to dietary changes preventing IBD but also achieve long-term disease control with good clinical outcomes and quality of life. Furthermore, it can be expected to inform microbiota modulating therapeutic approaches through further dietary-related studies on IBD and a better understanding of the human gut ecosystem.

\section{Conflict of interest}

The authors declare no potential conflicts of interest.

See the article "Increasing incidence of inflammatory bowel disease in children and adolescents: significance of environmental factors" via https://doi.org/10.3345/cep.2019.00500.

\section{References}

1. Molodecky NA, Soon IS, Rabi DM, Ghali WA, Ferris M, Chernoff G, et al. Increasing incidence and prevalence of the inflammatory bowel diseases with time, based on systematic review. Gastroenterology 2012; 142:46-54.

2. Uniken Venema WT, Voskuil MD, Dijkstra G, Weersma RK, Festen EA. The genetic background of inflammatory bowel disease: from correlation to causality. J Pathol 2017;241:146-58.

3. Liu JZ, van Sommeren S, Huang H, Ng SC, Alberts R, Takahashi A, et al. Association analyses identify 38 susceptibility loci for inflammatory bowel disease and highlight shared genetic risk across populations. Nat Genet 2015;47:979-86.

4. Misra R, Faiz O, Munkholm P, Burisch J, Arebi N. Epidemiology of inflammatory bowel disease in racial and ethnic migrant groups. World $\mathrm{J}$ Gastroenterol 2018;24:424-37.

5. Park SW, Kang YK, Koh H, Kim S. Increasing incidence of inflammatory bowel disease in children and adolescents: significance of environmental factors. Clin Exp Pediatr 2020;63:337-44.

6. Levine A, Sigall Boneh R, Wine E. Evolving role of diet in the pathogenesis and treatment of inflammatory bowel diseases. Gut 2018;67:1726-38.

7. Silva JPB, Navegantes-Lima KC, Oliveira ALB, Rodrigues DVS, Gaspar SLF, Monteiro VVS, et al. Protective mechanisms of butyrate on inflammatory bowel disease. Curr Pharm Des 2018;24:4154-66.

8. Hou JK, Abraham B, El-Serag H. Dietary intake and risk of developing inflammatory bowel disease: a systematic review of the literature. Am J Gastroenterol 2011;106:563-73.

9. Gu P, Feagins LA. Dining with inflammatory bowel disease: a review of the literature on diet in the pathogenesis and management of IBD. Inflamm Bowel Dis 2020;26:181-91.

10. Levine A, Wine E, Assa A, Sigall Boneh R, Shaoul R, Kori M, et al. Crohn's disease exclusion diet plus partial enteral nutrition induces sustained remission in a randomized controlled trial. Gastroenterology 2019;157: 440-50.

11. Kaenkumchorn T, Kesavan A. Dietary management of pediatric inflammatory bowel disease. J Med Food 2019;22:1092-9. 